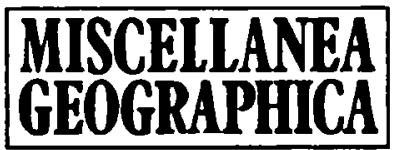

WARSZAWA 1994 Vol. 6

Joanna Widy-Kwiatkowska

\title{
L'ENVIRONNEMENT NATUREL ET LA BASE TOURISTIQUE EN ANDALOUSIE
}

Le tourisme exerce une influence point négligeable sur l'économie d'un côté et l'environnement naturel de l'autre, dans de nombreux pays. Les phénomènes sociaux économiques et écologiques qui l'accompagnent font l'objet d'études effectuées par des représentants de divers domaines de la science. De plus en plus souvent, les relations entre le tourisme et l'environnement naturel attirent l'attention des chercheurs.

La communication présente se pose pour but d'indiquer ces éléments de la nature dont l'influence sur la localisation et le développement de la base touristique en Andalousie est la plus grande.

D’après J.Warszyńska et A.Jackowski (1978), les principales caractéristiques de l'environnement naturel sont les suivantes:

- le relief,

- le réseau hydrographique

- la moyenne mensuelle de la température en juillet,

- la moyenne mensuelle des jours de soleil en juillet,

- la moyenne des précipitations en juillet,

- la distance de la mer,

- les forêts dont les forêts protégées.

On a adopté les capacités d'hébergement comme indice synthétique de l'aménagement touristique, et les analyses comprennent les structures d'accueil indiquées dans les cartes routières et touristiques de la région. L'analyse s'étend sur la vingtaine d'années, de 1970 à 1990. On a adopté l'analyse cartographique en tant que méthode de recherche.

L'étude effectuée a permis de constater une influence aussi bien des traits particuliers que celle de l'environnement naturel dans son ensemble sur la localisation de la base touristique dans un territoire donné ainsi que sur le développement de celle-ci au cours de la dernière vingtaine d'années. On a comparé la densité du réseau de structures d'accueil de la base touristique dans les territoires à la valeur définie d'une caractéristique choisie de l'environnement naturel, avec la densité moyenne desdites structures en Andalousie. On a eu recours à la formule suivante: 


\section{$\frac{100 \% \text { de la base touristique en Andalousie }}{100 \% \text { de la superficie de l'Andalousie }}=1$}

On peut supposer que la distance de la mer ne dépassant pas $10 \mathrm{~km}$ est un élément le plus important dont on tient compte lors de la localisation des structures d'accueil. L'aménagement dans ces terrains dépasse de sept fois la moyenne d'Andalousie qui, dans les années soixante-dix, s'élevait à 6 structures d'accueil sur $10 \mathrm{~km}^{2}$ et aujourd'hui - à 13 structures sur $10000 \mathrm{~km}^{2}$.

Dans d'autres territoires, la base est localisée de façon plus au moins égale et sa densité est deux fois moins grande que sur le littoral, résultat des attraits qu'offre la côte méditerranéenne. C'est une bande de plages de sable qui ne finissent pas, bordées de forêts, entrecoupées d'embouchures de fleuves. De ces plages, on aperçoit les hautes cimes $(3,300 \mathrm{~m})$ de la Sierra Nevada. Les territoires en question sont très peuplés et peuvent se prévaloir d'une histoire et des traditions vives et intéressantes.

Un autre élément qui, d'après les résultats des recherches, intervient au moment de la localisation de la base touristique est le climat et, en particulier ses caractéristiques suivantes: la moyenne de la température en juillet ne dépassant pas $25^{\circ} \mathrm{C}$, la moyenne des jours de soleil en juillet dépassant 27 , enfin la moyenne des précipitations en juillet ne dépassant pas $1 \mathrm{~mm}$. Ceci nous permet de constater que les endroits les plus attrayants à y passer les vacances se trouvent là, où il y a toujours du soleil, mais en même temps les températures sont relativement basses pour la zone du climat méditerranéen. Ainsi, ce sont les conditions idéales pour les vacances, surtout si l'on se rappelle que la moyenne de la température de la mer en juillet est d'env. $24^{\circ} \mathrm{C}$.

Passons maintenant au relief. Au point de vue de l'aménagement touristique, aussi bien les plaines que la haute montagne ont une importance considérable. C'est dans les plaines que se concentrent trois fois plus de structures d'accueil que la moyenne du territoire étudié. Ceci résulte du fait qu'il s'agit là d'un littoral, à quoi s'ajoute la facilité de construire dans les plaines, celles-ci, en même temps, se prêtant à l'installation d'un réseau des transports et à d'autres aménagements. Un autre facteur favorisant le développement de la base touristique ce seraient de grandes vallées des fleuves et rivières où on peut être certain de pouvoir s'approvisionner en eau.

Quant à la haute montagne, c'est un terrain offrant de nombreux attraits touristiques, ainsi il n'est pas étonnant que les indices de l'aménagement touristique y sont élevés et dépassent 3,7 fois l'aménagement moyen de la région. La Sierra Nevada qui se situe en deuxième place en Europe au point de vue de la hauteur se caractérise par un relief accidenté et par la possibilité de pratiquer les sports d'hiver pendant toute l'année - le touriste peut y faire du ski en maillot de bain, ensuite, il se retrouve au bord de la mer après un trajet en voiture qui ne dure qu'une haure; il peut se promener sur des glaciers ou emprunter des pistes de haute montagne. Ce sont les arguments qui mettent en évidence l'attrait incontestable de cette région. 
Le réseau hydrographique est un autre élément important pour l'aménagement touristique. Plus de 50\% de la base étant localisés dans les vallées de fleuves, l'aménagement touristique y dépasse deux fois la moyenne d'Andalousie. La connaissance du caractère spécifique du réseau hydrographique andalou, et surtout celle de son régime des eaux permet de constater que cet état des choses est dû moins au pittoresque de la région qu'aux facilités d'ordre organisationnel. Les fleuves et rivières alimentés principalement en hiver, en été conduisent moins d'eau, certains même se dessèchent complètement. Heureusement, il y a des eaux souterraines.

A la dernière place au point de vue de la localisation des structures d'accueil se situent les aires forestières. Il est vrai que les indices y dépassent la moyenne de la région, mais la différence n'est pas importante. La moyenne de l'aménagement y atteint 1,7 de la moyenne de la région. Les forêts ne sont qu'un supplément réhaussant les valeurs de la région et ne constituent pas une concurrence à la mer. C'est un élément supplémentaire, jamais principal.

Il est donc bien évident que l'environnement naturel exerce une influence non négligeable sur la localisation de la base touristique, mais peut-il influer également sur son développement?

Dans la communication présente, nous avons adopté l'accroissement du nombre de structures d'accueil en tant que développement de la base touristique. Cette simplification, adoptée après W.Gaworecki (1982) et A.Kornak (1985), nous paraît nécessaire à l'étude du problème qui nous intéresse, les matériaux accessibles ne permettant pas d'examiner le développement dans son aspect aussi bien quantitatif que qualitatif. Au cours de la période étudiée, l'accroissement du nombre de structures d'accueil était de 2,2 (ayant passé de 52 à 114). Cet accroissement était le plus important dans les hauts plateaux où le nombre de structures a augmenté 5,5 fois. La raison en pouvait être l'insuffisance d'endroits disponibles dans les plaines, de même que le voisinage immédiat de la mer, dans le Sud-Est andalou. Les terrains montagneux couvrant $26 \%$ de la superficie de la région, théoriquement toute la quatrième structure aurait dû y être localisée, mais comme les indices oscillent entre 0,2 et 0,4 de l'aménagement moyen de la région en question, il ne s'y trouve que toute la douzième structure. Il est possible que la cause en est le niveau d'investissements insuffisant. A la deuxième place du point de vue du développement de la base touristique se trouvent les grandes villes situées au bord d'un fleuve ou d'une rivière. Là, l'accroissement dépassait la moyenne de 1,2 fois et était probablement dû aux valeurs anthropogéniques et non aux fleuves eux-mêmes car dans d'autres territoires situés au bord de fleuves, le développement de la base touristique n'atteint pas la moyenne.

Les territoires éloignés de la mer plus de $40 \mathrm{~km}$ se caractérisent par une valeur importante de l'indice de développement. Le nombre de structures d'accueil y est passé de 10 à 25 durant la dernière vingtaine d'années. Ce 
Tableau 1

Le quotient du nombre de structures d'accueil de la base touristique, en 1970 et 1990

\begin{tabular}{|c|c|c|c|c|}
\hline \multirow{2}{*}{\multicolumn{2}{|c|}{ Situation des structures d'accueil }} & \multicolumn{2}{|c|}{ Nombre de structures } & \multirow[t]{2}{*}{ Quotient } \\
\hline & & 1970 & 1990 & \\
\hline \multicolumn{2}{|c|}{ Structures d'accueil au total } & 52 & 114 & 2,2 \\
\hline 1. & \begin{tabular}{|l} 
Relief \\
plaines \\
hauts plateaux \\
montagne \\
haute montagne
\end{tabular} & $\begin{array}{r}36 \\
2 \\
8 \\
6\end{array}$ & $\begin{array}{r}87 \\
11 \\
6 \\
10\end{array}$ & $\begin{array}{l}2,4 \\
5,5 \\
0,8 \\
1,7\end{array}$ \\
\hline 2. & $\begin{array}{l}\text { Eaux } \\
\text { sur les cours d'eau } \\
\text { en ville } \\
\text { a l'embouchure }\end{array}$ & $\begin{array}{l}28 \\
13 \\
10\end{array}$ & $\begin{array}{l}59 \\
23 \\
19\end{array}$ & $\begin{array}{l}2,1 \\
1,0 \\
1,9\end{array}$ \\
\hline 3. & $\begin{array}{l}\text { Climat } \\
\text { températures } \\
\text { jusqu'à } 25^{\circ} \mathrm{C} \\
\text { jusqu'à } 26^{\circ} \mathrm{C} \\
\text { plus de } 26^{\circ} \mathrm{C} \\
\text { nombre de jours de soleil } \\
\text { jusqu'à } 27 \\
\text { plus de } 27 \\
\text { précipitations } \\
\text { jusqu'à } 1 \mathrm{~mm} \\
\text { jusqu'à } 3 \mathrm{~mm} \\
\text { plus de } 3 \mathrm{~mm} \\
\end{array}$ & $\begin{array}{r}24 \\
20 \\
8 \\
8 \\
44 \\
13 \\
30 \\
9\end{array}$ & $\begin{array}{l}40 \\
42 \\
24 \\
\\
16 \\
98 \\
\\
31 \\
66 \\
17\end{array}$ & $\begin{array}{l}2,0 \\
2,1 \\
3,0 \\
\\
2,0 \\
2,2 \\
\\
2,4 \\
2,2 \\
1,9\end{array}$ \\
\hline 4. & $\begin{array}{c}\text { Distance de la mer } \\
\text { jusqu'à } 10 \mathrm{~km} \\
\text { jusqu'à } 20 \mathrm{~km} \\
\text { jusqu'à } 30 \mathrm{~km} \\
\text { jusqu'à } 40 \mathrm{~km} \\
\text { jusqu'à } 50 \mathrm{~km} \\
\text { plus de } 50 \mathrm{~km}\end{array}$ & $\begin{array}{r}29 \\
2 \\
2 \\
9 \\
2 \\
8\end{array}$ & $\begin{array}{r}61 \\
4 \\
4 \\
20 \\
6 \\
19\end{array}$ & $\begin{array}{l}2,1 \\
2,0 \\
2,0 \\
2,2 \\
3,0 \\
2,4\end{array}$ \\
\hline 5. & $\begin{array}{l}\text { Aires forestières } \\
\text { forêts } \\
\text { forêts protégées }\end{array}$ & $\begin{array}{r}39 \\
34 \\
5\end{array}$ & $\begin{array}{l}79 \\
68 \\
11\end{array}$ & $\begin{array}{l}2,0 \\
2,0 \\
2,2\end{array}$ \\
\hline
\end{tabular}

fait doit rester en rapport avec l'incidence de grandes villes dont la distance de la mer est justement de $40 \mathrm{~km}$. Citons enfin le climat - la moyenne mensuelle dépassant en juillet $26^{\circ} \mathrm{C}$ influe positivement sur le développement de la base touristique, sans oublier d'autres éléments attractifs, étrangers à la nature même. 
Tableau 2

Les Indices de l'aménagement touristique en Andalousie, en 1970 et 1990

\begin{tabular}{|c|c|c|c|}
\hline \multicolumn{2}{|r|}{ Éléments de l'environnement naturel } & \multicolumn{2}{|c|}{ Indices de l'aménagement } \\
\hline & & 1970 & 1990 \\
\hline 1. & $\begin{array}{l}\text { Relief } \\
\text { a. plaines jusqu'à } 100 \mathrm{~m} \\
\text { b. plaines, au total } \\
\text { c. hauts plateaux } \\
\text { d. montagne, au total } \\
\text { e.haute montagne, de plus de } 1500 \mathrm{~m}\end{array}$ & $\begin{array}{l}2,6 \\
2,9 \\
0,2 \\
0,5 \\
3,7\end{array}$ & $\begin{array}{l}2,9 \\
3,2 \\
0,4 \\
0,3 \\
2,8\end{array}$ \\
\hline 2. & $\begin{array}{l}\text { Réseau hydrographique } \\
\text { sur les fleuves et rivières, au total }\end{array}$ & 2,2 & 2,1 \\
\hline 3. & $\begin{array}{l}\text { Climat } \\
\text { a. température moyenne en juillet } \\
\text { jusqu'à } 25^{\circ} \mathrm{C} \\
\text { jusqu'à } 26^{\circ} \mathrm{C} \\
\text { plus de } 26^{\circ} \mathrm{C} \\
\text { b. nombre moyen de jours de soleil en juillet } \\
\text { jusqu'à } 27 \\
\text { plus de } 27 \\
\text { c. précipitations moyennes en juillet } \\
\text { jusqu'à } 1 \mathrm{~mm} \\
\text { jusqu'à } 3 \mathrm{~mm} \\
\text { plus de } 3 \mathrm{~mm} \\
\end{array}$ & $\begin{array}{l}0,3 \\
1,6 \\
3,1 \\
0,7 \\
2,0\end{array}$ & $\begin{array}{l}0,3 \\
1,6 \\
3,4 \\
0,7 \\
1,7\end{array}$ \\
\hline 4. & $\begin{array}{c}\text { Distance de la mer } \\
\text { jusqu'à } 10 \mathrm{~km} \\
\text { jusqu'à } 20 \mathrm{~km} \\
\text { jusqu'à } 30 \mathrm{~km} \\
\text { jusqu'à } 40 \mathrm{~km} \\
\text { jusqu'à } 50 \mathrm{~km} \\
\text { plus de } 50 \mathrm{~km}\end{array}$ & $\begin{array}{l}7,3 \\
0,5 \\
0,5 \\
2,1 \\
0,5 \\
0,5\end{array}$ & $\begin{array}{l}7,0 \\
0,5 \\
0,5 \\
2,2 \\
0,6 \\
0,5\end{array}$ \\
\hline 5. & $\begin{array}{l}\text { Aires forestières dont forêts protégées } \\
\text { a. forêts } \\
\text { b. forêts protégées }\end{array}$ & $\begin{array}{l}1,6 \\
2,4 \\
2,3\end{array}$ & $\begin{array}{l}1,7 \\
2,4 \\
2,5\end{array}$ \\
\hline
\end{tabular}

En récapitulant, nous pouvons constater que l'environnement naturel exerce une influence assez importante sur la localisation de la base touristique, cependant cette influence subit aujourd'hui des changements. De plus en plus souvent, les valeurs anthropogéniques modifient le rôle et l'ampleur de cette influence, jusqu'à présent dominants, de l'environnement naturel. 
BIBLIOGRAPHIE

Gaworecki W., 1978, Ekonomika i organizacja turystyki (Economie et organisation du tourisme), PWN, Warszawa.

Junta de Andalucia, "Andalucia '71", Direccion General de Turismo, Echelle au 1:800 000.

Junta de Andalucia, "Andalucia '89", Direccion General de Turismo, Echelle au 1:800 000.

Kornak. A., 1985, Ekonomika turystyki (Économie du tourisme), PWN. Warszawa.

Warszyńska J., Jack owski A., 1978, Podslawy geografii turyzmu (Les Rudiments de la géographie du tourisme), PWN, Warszawa. 